3

4 Shengli Tao ${ }^{\mathrm{a}, \mathrm{b}}$, Fangfang $\mathrm{Wu}^{\mathrm{a}}$, Qinghua $\mathrm{Guo}^{\mathrm{a}, \mathrm{c}^{*}}$, Yongcai Wang ${ }^{\mathrm{a}}$, Wenkai $\mathrm{Li}^{\mathrm{d}}$, Baolin Xue , 5 Xueyang $\mathrm{Hu}^{\mathrm{b}}$, Peng $\mathrm{Li}^{\mathrm{b}}$, Di Tian ${ }^{\mathrm{b}}$, Chao $\mathrm{Li}^{\mathrm{b}}$, Hui Yao ${ }^{\mathrm{b}}$, Yumei $\mathrm{Li}^{\mathrm{a}}$, Guangcai $\mathrm{Xu}^{\mathrm{a}}$, Jingyun $6 \quad$ Fang $^{\mathrm{b}}$

7

8 9

\section{Segmenting tree crowns from terrestrial and mobile LiDAR data by}

\section{exploring ecological theories}

\section{3}

(1)

8 a State Key Laboratory of Vegetation and Environmental Change, Institute of Botany, Chinese

Academy of Sciences, Beijing 100093, China

bepartment of Ecology, College of Urban and Environmental Sciences, and Key Laboratory

1 for Earth Surface Processes of the Ministry of Education, Peking University, Beijing

100871, China

'Sierra Nevada Research Institute, School of Engineering, University of California at Merced, Merced, CA 95343, USA

${ }^{\mathrm{d}}$ Guangdong Provincial Key Laboratory of Urbanization and Geo-simulation, School of Geography and Planning, Sun Yat-sen University, Guangzhou 510275, China 17

\footnotetext{
* Corresponding author.
}

Qinghua Guo

Sierra Nevada Research Institute, School of Engineering, University of California at Merced, 1 CA 95343, USA

Phone: (209) 228-2911

Fax: (209) 228-4047

4 Email: guo.qinghua@gmail.com 
Abstract - The rapid development of light detection and ranging (LiDAR) techniques is advancing ecological and forest research. During the last decade, numerous single tree segmentation techniques have been developed using airborne LiDAR data. However, accurate crown segmentation using terrestrial or mobile LiDAR data, which is an essential prerequisite for extracting branch level forest characteristics, is still challenging mainly because of the difficulties posed by tree crown intersection and irregular crown shape. In the current work, we developed a comparative shortest-path algorithm (CSP) for segmenting tree crowns scanned using terrestrial (T)-LiDAR and mobile LiDAR. The algorithm consists of two steps, namely trunk detection and subsequent crown segmentation, with the latter inspired by the well-proved metabolic ecology theory and the ecological fact that vascular plants tend to minimize the transferring distance to the root. We tested the algorithm on mobile-LiDAR-scanned roadside trees and T-LiDAR-scanned broadleaved and coniferous forests in China. Point-level quantitative assessments of the segmentation results showed that for mobile-LiDAR-scanned roadside trees, all the points were classified to their corresponding trees correctly, and for T-LiDAR-scanned broadleaved and coniferous forests, kappa coefficients ranging from 0.83 to 0.93 were obtained. We believe that our algorithm will make a contribution to solving the problem of crown segmentation in T-LiDAR scanned-forests, and might be of interest to researchers in LiDAR data processing and to forest ecologists. In addition, our research highlights the advantages of using ecological theories as guidelines for processing LiDAR data. 
47 Keywords terrestrial LiDAR; segmentation; shortest path; mobile LiDAR; metabolic ecology 48 theory; DBSCAN

49 


\section{Introduction}

51 Light detection and ranging (LiDAR) is a state-of-the-art active remote-sensing technique

52

53

capable of capturing three-dimensional (3D) measurements by emitting laser light (Wehr and Lohr, 1999). In recent decades, LiDAR from different platforms, i.e., spaceborne, airborne, terrestrial, and mobile LiDAR, has shown great utility in estimating biophysical forest properties, including canopy height (Naesset 1997), forest biomass (Zhao et al., 2009; Li et al., 2015), and high-resolution carbon stock (Asner et al., 2010), because of its ability to record vertical structures on spatial scales ranging from individual trees to landscape.

Of all the LiDAR platforms available, airborne LiDAR is increasingly providing stand or tree characteristics (mean tree height, tree number, and individual tree height) over large areas (Lovell et al., 2011), but at the cost of missing details at branch level (Dassot et al., 2011). Airborne LiDAR also fails to detect small trees in lower canopy layer (Maltamo et al., 2004). As a complement to airborne LiDAR, terrestrial LiDAR (T-LiDAR) and mobile LiDAR operate underneath the canopy, and are therefore able to acquire dense point clouds recording detailed branch structures (Figure 1), for both canopy and overtopped trees in sub-canopy layers. In particular, T-LiDAR and mobile LiDAR shed new light on forest structural measurements for which traditional approaches remain time-consuming and destructive (Dassot et al., 2011). For example, the 3D structural information obtained using T-LiDAR enables tree volume metrics to be calculated by mathematical integration instead of section measurement methods (Lefsky and McHale, 2008; Dassot et al., 2012). Branch-level information including branch length, branch angle can be obtained using T-LiDAR and morphologically analyzed to explore branch networks and the structural complexity of a 

estimate the fluxes of carbon, water, and energy between the atmosphere and trees (Zheng et

74

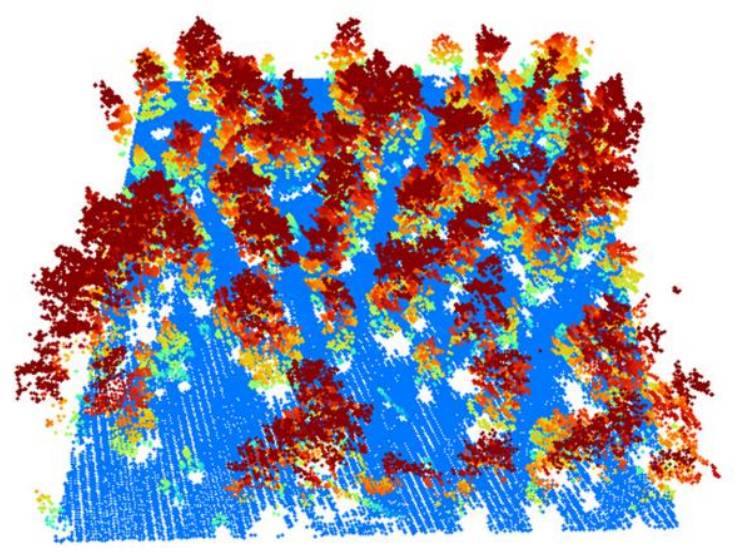

79 through 3D visualization (Xu et al., 2007).

\section{8} indicate different height levels. al., 2013), can be accurately estimated using leaf points of individual trees scanned using T-LiDAR. Moreover, discrete points scanned using T-LiDAR and mobile LiDAR can be reconstructed into tree models, enabling computer simulation of forests, and public education

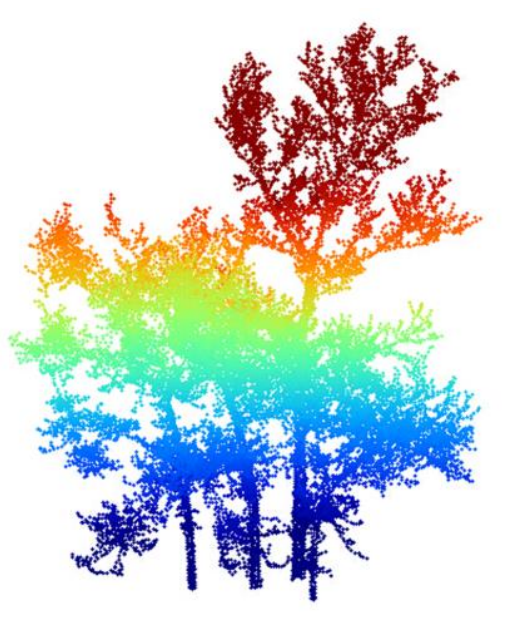

B

Figure 1. Comparison of airborne LiDAR data (A, scanned by the National Critical Zone Observatory Project) and terrestrial LiDAR (T-LiDAR) data (B). This figure shows that airborne LiDAR data can provide basic forest characteristics like tree locations and tree heights, whereas T-LiDAR data can provide detailed information at branch level such as branch angle, branch size and branch length. Accurate crown segmentation of T-LiDAR-scanned forests will therefore greatly benefit the quantification of tree branching networks. But the crown shape of trees in a forest can be extremely irregular and intersected (Figure 1B), posing great challenges for crown segmentation. Different colors in this figure 
The above-mentioned forest measurements and applications require accurate crown

91

segmentation from T-LiDAR or mobile LiDAR point cloud as an essential prerequisite

(Figure 1). Recent decades have witnessed the rapid development of single-tree segmentation algorithms, but most of these are based on airborne LiDAR data. Conventionally, trees are detected from Canopy Height Models (CHMs) which are interpolated from the first return points. Hyyppa et al. (2001) segmented individual trees from CHM images using a region-growing method on conifer stands. The continuing efforts of Hyyppa et al. (2012) suggest that trees can be better discriminated using last-return data than using first-return data. Chen et al. (2006) used marker-controlled watershed segmentation for identifying individual trees in savanna woodlands. Popescu et al. (2002) used local filtering techniques for segmenting trees from deciduous, coniferous, and mixed stands, with the local maxima found by a round window resembling a tree crown, rather than a square one. Recently, more advanced tree detection techniques operating directly on point clouds have been developed. Reitberger et al. (2009) and Yao et al. (2012) used a normalized cut method for tree segmentation from waveform LiDAR data. Li et al. (2012) developed a top-down region-growing point cloud segmentation (PCS) method for segmenting complex mixed forests. PCS obtained an overall accuracy of $94 \%$ in the Sierra National Forest, which was overwhelmingly superior to the traditional marker-controlled watershed segmentation (Tao et al., 2014).

Accurate individual tree crown segmentation using T-LiDAR or mobile LiDAR data is still challenging, especially for broadleaved trees of which tree crowns can be extremely 
111 irregular and are often heavily intersected (Figure 1B). Although there have been pioneering 112 studies on tree segmentation using T-LiDAR data or mobile LiDAR data, the focus tends to be 113 trunk detection (Simonse et al., 2003; Gorte and Pfeifer, 2004; Brolly and Kiraly, 2009;

114 Huang et al., 2011; Schilling et al., 2011; Olofsson et al., 2014), and tree crowns are often 115 simply approximated as a cylinder centralizing tree trunks or tree tops. For examples, Bienert et al. (2006) and Maas et al. (2008) automatically detected trunks from T-LiDAR point clouds,

117 and further approximated the crown part of a tree as a vertical cylinder with a predefined 118 radius surrounding the stem. Similarly, Livny et al. (2010) segmented roadside trees by 119 classifying points within a constant distance around local maxima into a single tree point cluster. Wu et al. (2013a) segmented roadside trees with high accuracy using a voxel-based marked-neighborhood-searching method. However, roadside trees are uniform in crown shape and usually not crown intersected, therefore previous methods for segmenting roadside trees might not be able to deal with forests exhibiting crown intersecting and irregular crown 124 shapes.

This paper proposes a new algorithm for segmenting individual tree trunks as well as crowns from both mobile-LiDAR-scanned roadside trees and T-LiDAR-scanned forest (including both broadleaved and coniferous forests). The newly proposed algorithm, namely the comparative shortest-path algorithm (CSP), was inspired by ecological basis and classical metabolic ecological theory (Leopold, 1971; West et al., 1999a). The development of the new algorithm is a basis for our future researches aiming at quantifying the branching network of trees. To give a clear illustration of the new algorithm, the rest of this paper is organized as 
133

detailed illustration of the algorithm in Section 3. To demonstrate the effectiveness of the comparative shortest-path algorithm, the PCS algorithm developed by Li et al. (2012) was performed on the same dataset and the results were compared with those obtained using the new algorithm. Sections 4 and 5 provide a comparison of the results and evaluate the two algorithms. Finally, conclusions are drawn in Section 6.

\section{Datasets}

Five datasets were used for testing our algorithm, including four T-LiDAR-scanned forest plots in China (Figure 2), and one mobile-LiDAR-scanned point cloud of roadside trees. In February 2014, during the leaf-off period, a Carya illinoinensis forest plot (Plot 1 hereafter) in the Beijing Botanical Garden was scanned using a Riegl VZ400 (Riegl Inc., Austria) T-LiDAR system mounted on a tripod (Table 1). In September 2014, during the leaf-on period, the plot was scanned a second time. In addition, a Pinus tabuliformis forest plot (Plot 2 hereafter) in Dongling Mountain in Beijing and a Castanopsis Sclerophylla forest plot (Plot 3 hereafter) in Anhui province in China were scanned using the same T-LiDAR system in December 2014 and January 2015, respectively (Figure 2). The sampling setup was distributed on a $10 \mathrm{~m} \times 10 \mathrm{~m}$ grid in each plot (Cifuentes et al., 2014). To ensure point density at the crown part, additional scanning towards forest canopy was also conducted. Plot 1 consists of trees of different sizes and irregular crown shapes (Figure 1B; Table 2). Small trees in this plot are overtopped by large trees, posing challenges for crown segmentation. Plot 2 is planted forest with a constant planting distance of ca. $1.6 \mathrm{~m}$, and is therefore heavily crown intersected. Plot 3 is a natural forest with not only intersecting canopy observed, but also 

and finally downsampled to keep an averaged point distance of $10 \mathrm{~cm}$ to reduce computational burden using RISCAN PRO software (Riegl Inc., Austria).
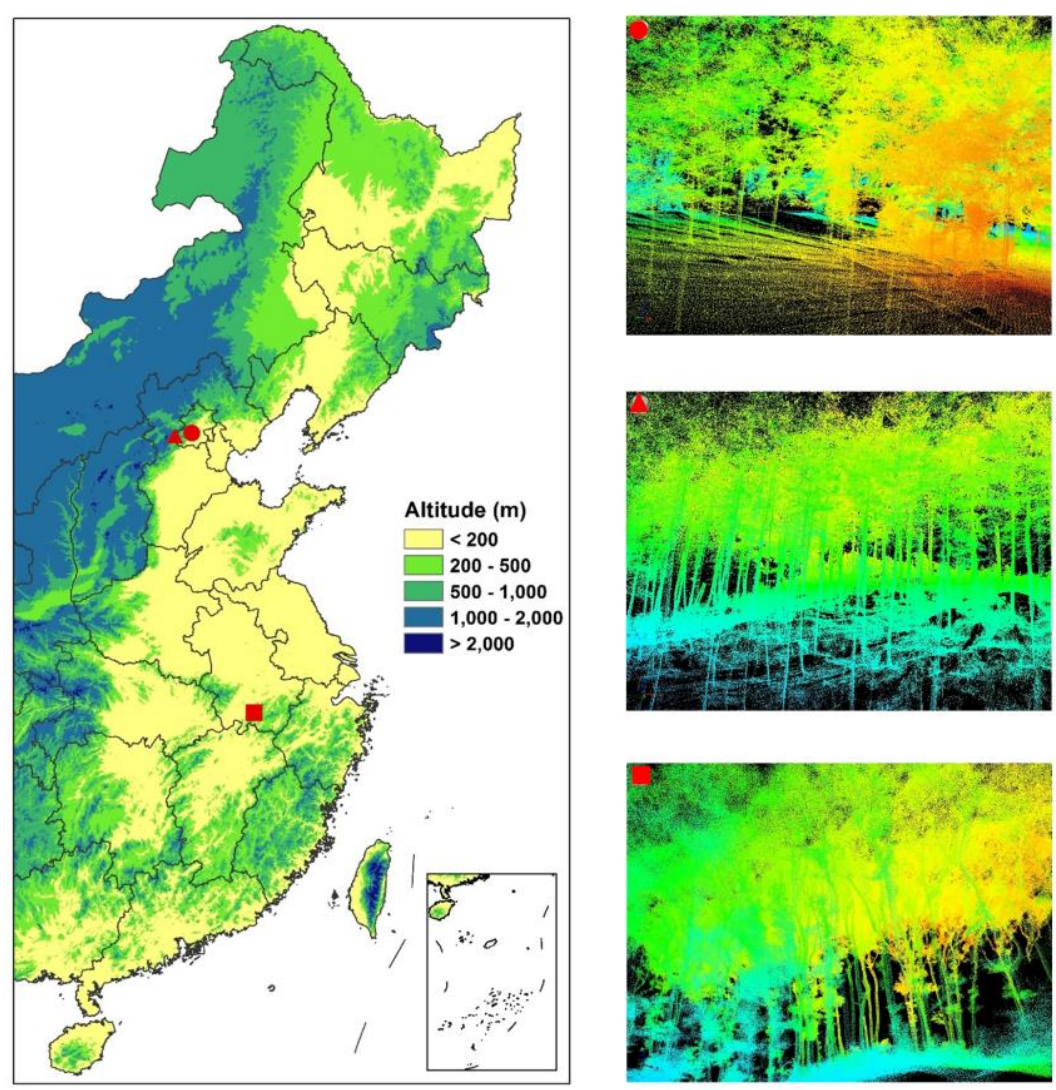

160 Figure 2. Forest plots scanned using Riegl VZ 400 T-LiDAR system and their point clouds for

161 testing the proposed new algorithm. The testing forest data includes Carya illinoinensis forest forest in Jitan, Anhui Province, China (bottom). 

system.

168

\begin{tabular}{lll}
\hline Riegl VZ-400 & Field of view & $\begin{array}{l}\text { Horizontal: } 0-360^{\circ} \\
\text { Vertical: }-40^{\circ} \text { to } 60^{\circ}\end{array}$ \\
& Effective measurement rate & Up to 122,000 measurements/s \\
& Detection range & $600 \mathrm{~m}$ at $90 \%$ reflectivity \\
& Laser beam divergence & $0.35 \mathrm{mrad}$ \\
& Scanning accuracy & $2 \mathrm{~mm}$ at $100 \mathrm{~m}$ distance \\
& Minimum range & $1.5 \mathrm{~m}$ \\
Laser wavelength & Near infrared \\
& Camera & Equipped with Nikon D300s \\
& Scanner weight & $9.8 \mathrm{~kg}$ \\
\hline Detection range & Up to $70 \mathrm{~m}$ \\
Scanning accuracy & $2 \mathrm{~cm}$ \\
Vehicle velocity & $20-60 \mathrm{~km} / \mathrm{h}$ \\
Scanning mode & Fixed direction \\
& Vertical scanning angle & $360^{\circ}$ \\
Points per second & Up to 700,000 \\
Scanner weight & $1 \mathrm{~kg}$ \\
\hline
\end{tabular}

169

170

171

172

173

174

175

176 
178 Table 2. Forest plots scanned using Riegl VZ400 T-LiDAR system for testing the proposed algorithm. The numbers inside parentheses are the 179 number of scans conducted toward forest canopy.

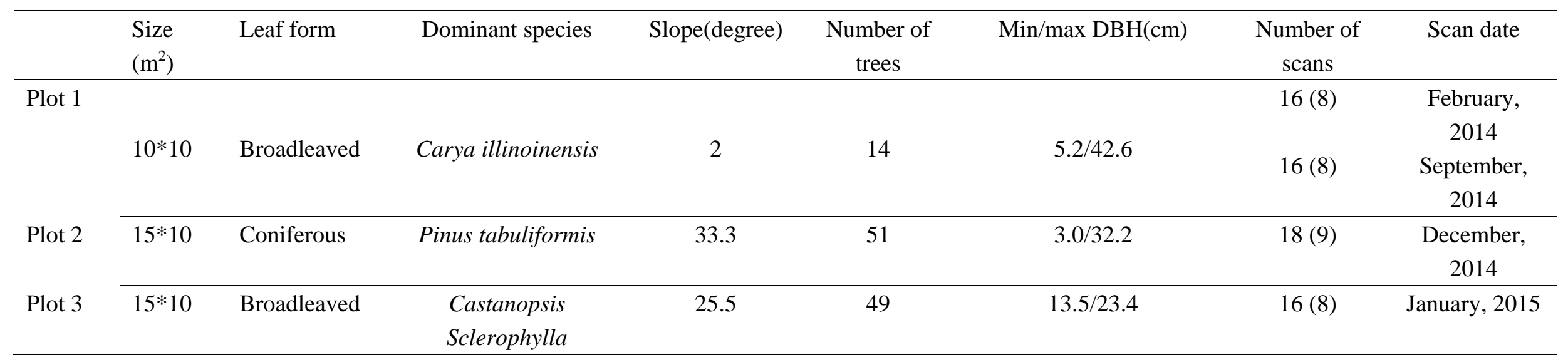


The roadside trees were scanned using a Li-mobile LiDAR system (LiForest Ltd., Co.) at

182

183

184

185

186

187

188

189

190

191

192

193

194

195

196

197 198 the Beijing Botanical Garden (Table 1). The mobile LiDAR system consisted of three major components: a Velodyne laser scanner, NovAtel Inertial Measurement Unit (IMU), and NovAtel high-precision Global Positioning System (GPS). The scanning angles of the laser scanner spanned a range up to $360^{\circ}$ and the angle resolution was $0.1^{\circ}$. The IMU measured and reported the vehicle's velocity, orientation, and gravitational forces. Together with the time information provided by the GPS, point clouds were generated automatically. The density of the point cloud depended on both the scanning speed of the laser scanner, which was up to 700,000 , and the velocity of the vehicle, which was around $25 \mathrm{~km} / \mathrm{h}$ in our case, resulting in clear recording of trees.

\section{Methodology}

\subsection{Comparative shortest-path algorithm (CSP)}

The strategy for individual tree segmentation consisted of three major parts: point cloud normalization, trunk detection and DBH estimation, and finally crown segmentation. In addition, we also performed PCS developed by Li et al. (2012) and compared the results with those obtained using the comparative shortest-path algorithm.

\section{Step 1: Point cloud normalization}

The point clouds were normalized before segmentation using the following formula:

$$
Z_{i}-Z_{i}^{\text {dem }}
$$


where $Z_{i}$ is the $z$-value of the $i$ th point, and $Z_{i}^{\text {dem }}$ is the corresponding value of the Digital

Elevation Model (DEM) grid onto which the point is projected. The DEM was interpolated using ordinary kriging method at $0.2 \mathrm{~m}$ resolution (Guo et al., 2010), with the lowest points in each $0.25 \mathrm{~m}^{2}$ grid treated as ground points (McDaniel et al. 2012). After normalization, the elevation value of each point indicates the height from zero (ground) to the point (Li et al., 2012). It is worth mentioning that point cloud normalization does not change the spatial 207 distribution of trees and branches.

\section{Step 2: Trunk detection and DBH estimation}

209 In contrast to forest canopy, which tends to intersect as a result of competition for light, trunks are naturally separated from each other and can therefore be accurately detected. In the current research, a cluster algorithm, namely Density-based spatial clustering of applications with noise (DBSCAN), was adopted for trunk segmentation, because it has the merits of robustness to noise points and efficiency (Wu et al., 2013b). DBSCAN is a density-based 214 clustering algorithm that does not require the number of clusters as an input parameter (as 215 opposed to the k-means algorithm). Instead, by defining the minimum number of points 216 required to form a cluster (MinPts) or the radius of the neighborhood (Eps-neighborhood of a 217 point), DBSCAN is able to detect clusters of arbitrary shape automatically. Starting with any 218 arbitrary point $p$ in point cloud $D$, the Eps-neighborhood of $p$, denoted by $N_{\text {Eps }}(p)$, is defined 219 as the set of points $q$ (including point $p$ itself) such that the distance between $p$ and $q$ is within 220 Eps:

$$
N_{\mathrm{Eps}}(p)=\{q \in D \mid \operatorname{dist}(p, q) \leq \mathrm{Eps}\}
$$


If there are enough points contained in that neighborhood, which means $\left|N_{\text {Eps }}(\mathrm{q})\right| \geq \operatorname{MinPts}$, a cluster is initiated; otherwise, $p$ is labeled as a noise point (Ester et al., 1996; Wu et al., 2013b). This procedure is repeated for each point that has not been visited. It is worth noting

225 226

that the two parameters required for DBSCAN, namely MinPts and Eps, need not to be provided at the same time. Eps can be calculated from the size of the point cloud and MinPts when the points exhibit a relatively uniform distribution, using the following equations (Daszykowski et al., 2002):

$$
\begin{aligned}
& \text { Eps }=\sqrt{\frac{T \cdot M i n P t s \cdot \Gamma[(1 / 2) \cdot n+1]}{m \sqrt{\pi^{n}}}} \\
& T=\prod_{i=1}^{n}\left\{\max \left(X_{i}\right)-\min \left(X_{i}\right)\right\}
\end{aligned}
$$

where $m$ denotes the number of points, $n$ is the dimensionality of the points, $\Gamma$ is the gamma function, $X$ is the point dataset, and $T$ is the volume of the experimental space formed by $m$ points.

DBSCAN is well suited to automatic trunk segmentation because the number of trunks in a plot is not available in advance. To apply DBSCAN to our plot, a point slice of vertical size of $10 \mathrm{~cm}$ cut from the point cloud at a height of $1.3 \mathrm{~m}$ was used as the input, and the parameter MinPts was set at 2. Figure 3A shows the trunks detected using DBSCAN, taking the T-LiDAR-scanned deciduous forest plot (Plot 1) as an example. After trunk segmentation, the size of each trunk was quantified and the DBH was measured from the points as follows: first, for each trunk slice, the gravity centers of all the points were calculated, and then radius was calculated as the mean distance from the weight center to the points forming the trunks (Haralick, 1974); finally, DBH was calculated from radius. If a point slice from $1.3 \mathrm{~m}$ was not 
244 The estimated DBH values from LiDAR points were highly correlated with ground-truth 245 measurements, indicating the effectiveness of the DBH-extraction method (Figure 3B).

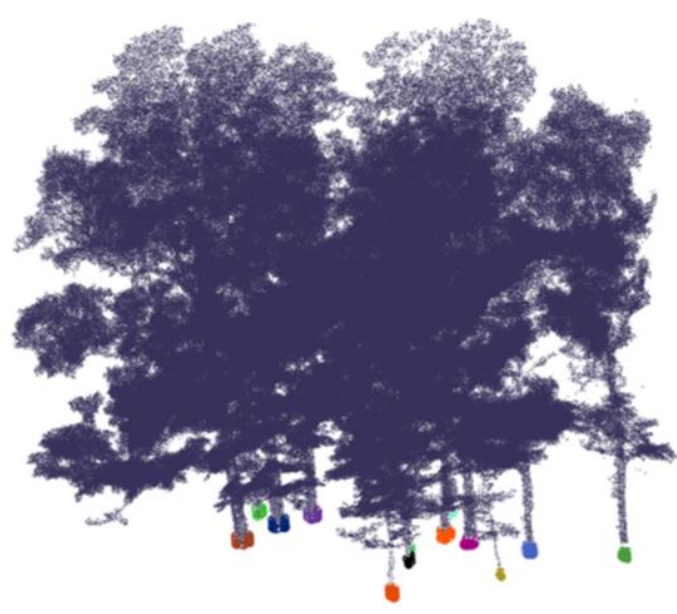

A

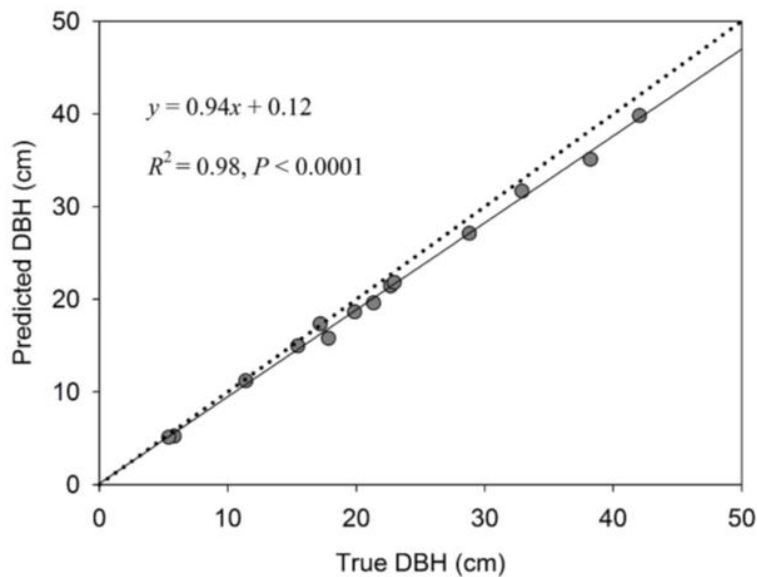

B

Figure 3. Trunk detection using DBSCAN (A) and accuracy of DBH estimation (B);

248 T-LiDAR-scanned broadleaved forest is used as an example. Trunks are indicated in different 249 colors.

252 The branch vascular structure of a tree is filled with conducting tubes for transferring water 253 and other nutrients, therefore "paths" can be imaged inside the vascular structure stretching 254 continuously from the roots to the leaves through the trunk, second-order branches, 255 lower-order branches, twigs, and petioles, successively. For trees that are space-sparsely 256 distributed with no crown intersection, the paths originating from the trunk of one tree to any 257 part of its crown do not reach other trees, because no route bridging the target trees to its neighbors is available (Figure 4A). The points in the paths originating from the trunk of the 

connect and intersect, paths will run continuously into neighboring trees intersecting with the target tree at the crown part. This poses a great challenge for crown segmentation. Fortunately, for the purpose of maximizing efficiency, the structural designs of trees have been shaped by evolution in a way that reduces the distances over which materials are transported (Leopold 1971; West et al., 1999a). Thus, according to this biological theory, if two trees of equal size are crown intersected, the points at the intersecting part probably belong to the tree to the 266 trunk of which a shorter transporting distance can be obtained (Figure 4B). For 267 crown-intersecting trees that differ greatly in size, a direct comparison between transporting distances is not appropriate, as shown in Figure 4C, in which points $v_{1}$ and $v_{2}$ belong to Tree 1 , 269 but the distances from $v_{1}$ and $v_{2}$ to Trunk 1 are longer than the distances to Trunk $k_{2}$. As a 270 consequence, part of Tree 1 will be falsely segmented to Tree2. In order to deal with this 271 problem, we can scale the transporting distance before comparison, using the metabolic 272 ecology theory (West et al., 1999b; Enquist, 2002; Bentley et al., 2013), which suggests the 273 existence of a universal power-law relationship between branch length and branch radius, 274 with a fixed scaling exponent close to $2 / 3$. In other words, the transporting distances of one 275 tree were scaled using the following equation, before comparing the transporting distances:

$$
D_{v \rightarrow \text { Trunk }}^{N}=D_{v \rightarrow \text { Trunk }} / \mathrm{DBH}^{2 / 3}
$$

277 where $D_{v \rightarrow \text { Trunk }}$ is the transporting distance from point $v$ to the tree trunk and $D^{N}{ }_{v \rightarrow \text { Trunk }}$ is the 278 DBH-scaled transporting distance. Note that DBH of each trunk was calculated in step 2. 

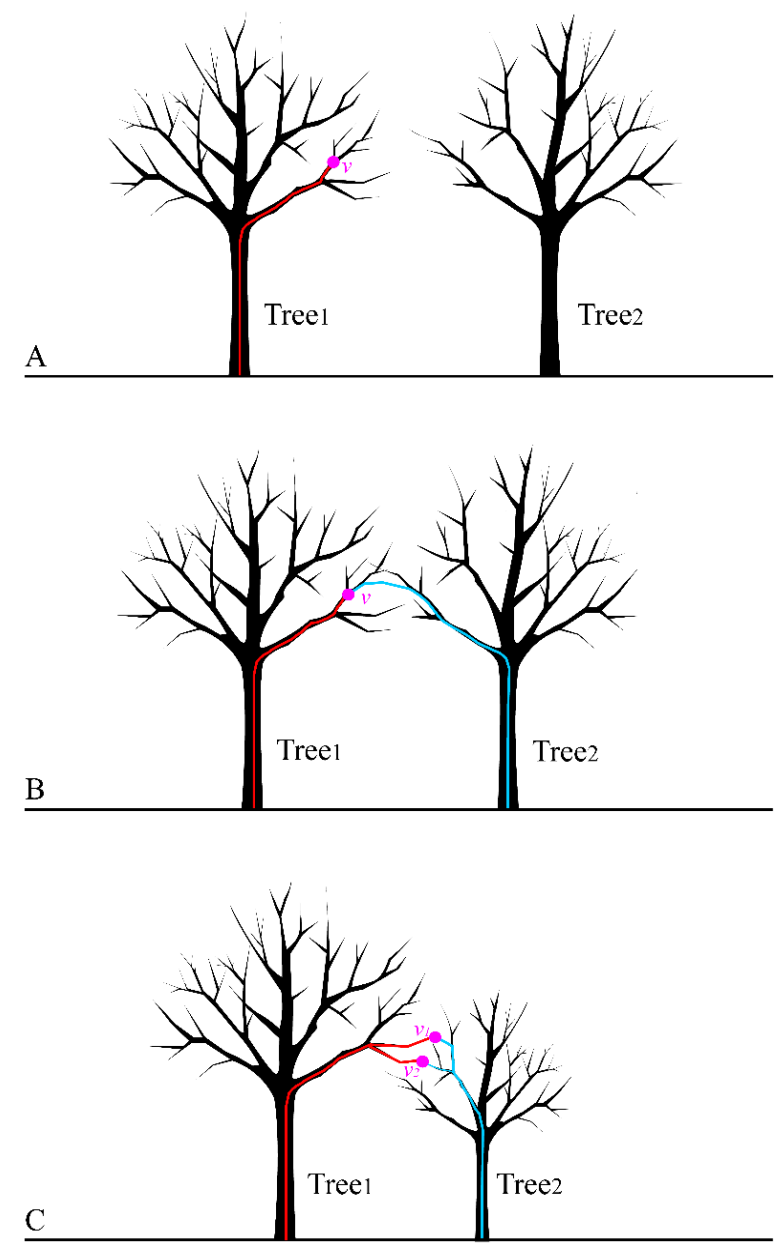

281 Figure 4. Conceptual images illustrating the comparative shortest-path algorithm (CSP), 282 taking two trees as an example. (A) Two trees are not crown intersected, and point $v$ (together 283 with other points in Tree 1 ) will be classified to Tree ${ }_{1}$, because path (indicated by the red line) 284 is only available to the root of Tree1; (B) two trees of equal size are crown intersected, and 285 point $v$ (together with other points in Tree 1 ) will still be classified to Tree 1 because path 286 distance to the root of Tree 1 is shorter than that to the root of Tree2; (C) two trees of different 287 size are crown intersected, and points $v_{1}$ and $v_{2}$ of Tree 1 will be falsely classified to Tree 2 if 288 the rule in Figure 4B is directly applied; to reduce errors, we therefore scaled the path 289 distances according to metabolic ecology theory before comparison of path distances. 
293 be classified into individual trees using the above-mentioned strategy, which is named as 294 comparative shortest-path strategy. Fortunately, the path and transporting distance can be 295 obtained using the shortest-path algorithm of graph theory, which has been extensively used by previous researchers for tree topology analysis (Xu et al., 2007; Livny et al., 2010;

297 Schilling et al., 2012). In short, the whole procedure can be summarized in the following steps:

298 1) establish a graph $G=(V, E)$ from the LiDAR point cloud of trees, where $V$ represents the 299 vertices (points of point cloud) and $E$ represents the edges (connection between two points); 2) calculate the shortest paths and DBH-scaled shortest paths using Dijkstra's shortest-path algorithm (Dijkstra, 1959); and 3) classify the points using the comparative shortest-path strategy.

1) To establish the graph $G$, LiDAR points were organized using a kd-tree structure, to 304 speed up the process. Then a range search was conducted to connect each point $v$ with its neighbors within a fixed 3D Euclidean distance.

307 Euclidean distance weights (Cormen et al., 2009), and a path $p$ as a sequence of vertices $p=$ $308\left(v_{1}, v_{2}, \ldots, v_{k}\right)$, such that each adjacent pair of vertices is connected by an edge, the weight of 309 path $p$ denoted as $w(p)$ is defined as sum of the weights of its constituent edges:

$$
w(p)=\sum_{i=1}^{k} w\left(v_{i-1}, v_{i}\right)
$$

311 The shortest-path problem can then be expressed as follows: starting from the source vertex $u$, 312 find the path to vertex $v$ under the constraint of minimizing the weight of the constituent 
313 edges $w(u, v)$ of path $p$. If there is no path stretching from vertex $u$ to vertex $v$, the weight of

314 this shortest path is treated as infinite. In summary, the shortest-path weight $w(p)$ is

$$
w(p)=w(u, v)=\left\{\begin{array}{cc}
\min \{w(p): u \stackrel{p}{\longrightarrow} v\} & \text { if there is a path from } u \text { to } v \\
\infty & \text { otherwise }
\end{array}\right.
$$

3) For trees that are space-sparsely distributed with no crown intersection as in the case of mobile-LiDAR-scanned roadside trees, points were classified as follows: let Tree 1 be the target tree, the trunk of Tree 1 be denoted by Trunk 1 , and let $D^{N}{ }_{v \rightarrow \text { Trunk1 }}$ symbolize the DBH-scaled shortest-path distance from point $v$ to Trunk1 (Figure 4A):

for each point $v \in V$ :

$$
\text { if } D_{v \rightarrow \text { Trunk } 1 \neq \infty}^{N}
$$

$v \in$ Tree $_{1}$

For trees with crowns that are heavily intersected, the above-mentioned comparative shortest-path strategy is used to classify the points in the crown-intersecting area (Figures 4B and 4C): if Tree 1 and Tree 2 are crown intersected, for point $v \in$ Tree $_{1}$ and Tree, we classify $v$ using the following rules:

$$
\begin{gathered}
\text { if } D_{v \rightarrow \text { Trunk1 }}^{N}<D^{N}{ }_{v \rightarrow \text { Trunk2: }} \\
\text { else } \quad \begin{array}{l}
v \in \text { Tree }_{1} \\
v \in \text { Tree }_{2}
\end{array}
\end{gathered}
$$

where $D^{N}{ }_{v \rightarrow \text { Trunk1 }}$ symbolizes the scaled shortest-path distance from point $v$ to the trunk of

Tree 1 and $D^{N}{ }_{v \rightarrow \text { Trunk2 }}$ symbolizes the scaled shortest-path distance from point $v$ to the trunk of

Tree2.

For clarity, we have presented examples containing only two trees to illustrate the simultaneously, the following pseudo codes can be used: 


\subsection{Point cloud segmentation}

The PCS algorithm developed by Li et al. (2012) was compared with the comparative

343

shortest-path algorithm. The core idea of the PCS is summarized as follows: treating the highest point in a point cluster as a tree top, each point below the tree top is judged using a spacing threshold rule to determine whether the point belongs to the tree initialized by the tree top. A single tree is segmented out after all points below the highest point have been judged, and this procedure is implemented iteratively for other trees, as shown in the following pseudo code:

\section{While $P$ is not empty:}

if point $a$ is a local maximum in two-dimensional circular space with a radius $R$ :

if $d \min 1>d$ : point $a$ belongs to $N i$;

if $d \min 1<=d$ and $d \min 1<d \min 2$ : point $a$ belongs to $T i$; if $d \min 1<=d$ and $d \min 1>d \min 2$ : point $a$ belongs to $N i$; else:

if $d \min 1<=d \min 2$ : points $a$ belongs to $T i$;

if $d \min 1>d \min 2$ : point $a$ belongs to $N i$;

$$
P=P-T i
$$

where $P$ denotes the point cloud, $T i$ denotes the target tree that will be segmented at the $i$ th time, $N i$ is an outlier set, dminl (or dmin2) denotes the minimum two-dimensional (2D) Euclidean distance from any point in $T i$ (or $N i$ ) to point $a$, and $d$ is an adaptive spacing threshold (Li et al., 2012; Tao et al., 2014).

\subsection{Implementation}

We used Matlab 2013b with the parameter Eps set at 2 for the DBSCAN procedure, to detect individual trunks from both T-LiDAR-scanned forest and mobile-LiDAR-scanned roadside trees. Each trunk (with its DBH) is assigned a unique ID. The shortest-path algorithm for 
crown segmentation was then implemented in $\mathrm{C}++$ using the boost graph library with a fixed neighbor search radius of $30 \mathrm{~cm}$ for all the testing point clouds (Siek et al., 2001). Point classified to a trunk will be labeled with the same ID with its belonging trunk. The entire procedure can thus be imaged as growing each trunk into a tree by finding the points closer to

370 it than to neighboring trunks in terms of shortest-path distance. For mobile-LiDAR-scanned 371 roadside trees, the shortest paths were not scaled, in order to reduce the computational burden.

375 were inverted (i.e., rotated 180 degrees) before the implementation of PCS to achieve better segmentation accuracy.

\subsection{Accuracy assessment}

378 We assessed the segmentation results at not only tree level, but also point level. For tree level

379 assessment, if a tree exists and is segmented out from the point cloud, it is called true positive 380 (TP); if a tree is not segmented but assigned to a nearby tree, it is called false negative (FN); if 381 a tree does not exist but is segmented from the point cloud, it is called false positive (FP). 382 High TP value and low FN and FP values correspond to high accuracy. To evaluate the 383 accuracy at tree level, we calculated recall $(r)$, precision $(p)$, and $F$-score using the following equations (Goutte and Gaussier 2005; Sokolova et al., 2006):

$$
F=2 \times \frac{r \times p}{r+p}
$$

$$
\text { where } r=\frac{T P}{T P+F N} \text { and } p=\frac{T P}{T P+F P}
$$



trees) and false negative points (points segmented to other trees) were identified and falsely segmented points) and Cohen's kappa coefficient based on the error matrix to assess the segmentation accuracy at point level.

\section{Results}

Figure 5 shows the segmentation results for mobile-LiDAR-scanned roadside trees obtained using the comparative shortest-path algorithm (Figure 5A) and PCS (Figure 5B). Visual inspection indicates that both the algorithms succeed in segmenting roadside trees exhibiting no crown intersections, with no false segmentation observed. Figure 6 and supplementary video show the segmentation results for the T-LiDAR-scanned forest plots, obtained using the comparative shortest-path algorithm (A-D) and PCS (E-H); the accuracy assessments of the segmentation results at tree level are shown in Table 3. Individual trees in Plot 1(both leaf-off and leaf-on conditions) and Plot 2 were correctly segmented from the point cloud by the

404 comparative shortest-path algorithm, but for Plot 3, four trees were falsely segmented because 405 of trunk occlusion (Table 3; Figure 6; supplementary video). In contrast, much more trees were falsely segmented by PCS, including four false-positive and four false negative trees for

407 Plot 1, 15 false-positive and 12 false negative trees for Plot 2, and one false-positive and 13 408 false negative trees for Plot 3 (Table 3; Figure 6; supplementary video). 

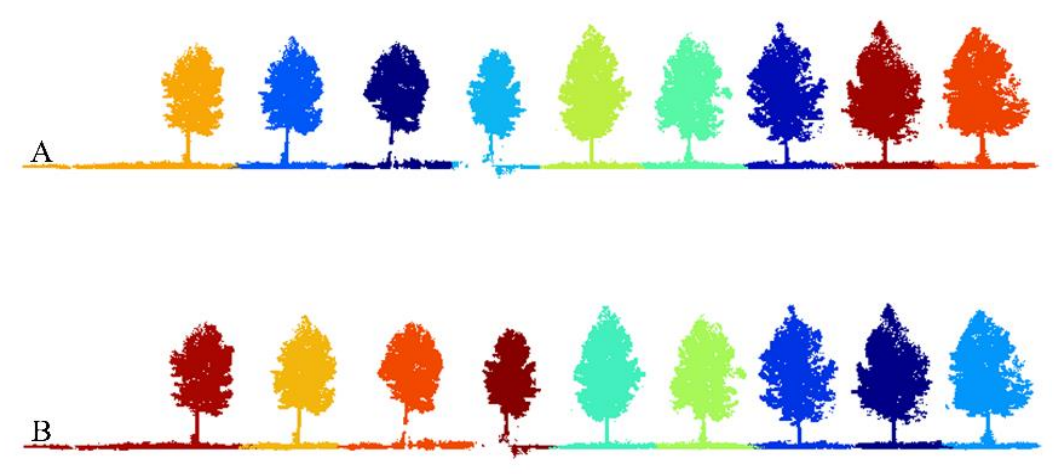

410

411 Figure 5. Segmentation results for mobile LiDAR scanned roadside trees using comparative

412 shortest-path algorithm (A) and PCS algorithm (B). This figure indicates that both algorithms

413 are able to segment trees that are not crown intersected. Different colors indicate different 414 trees. 

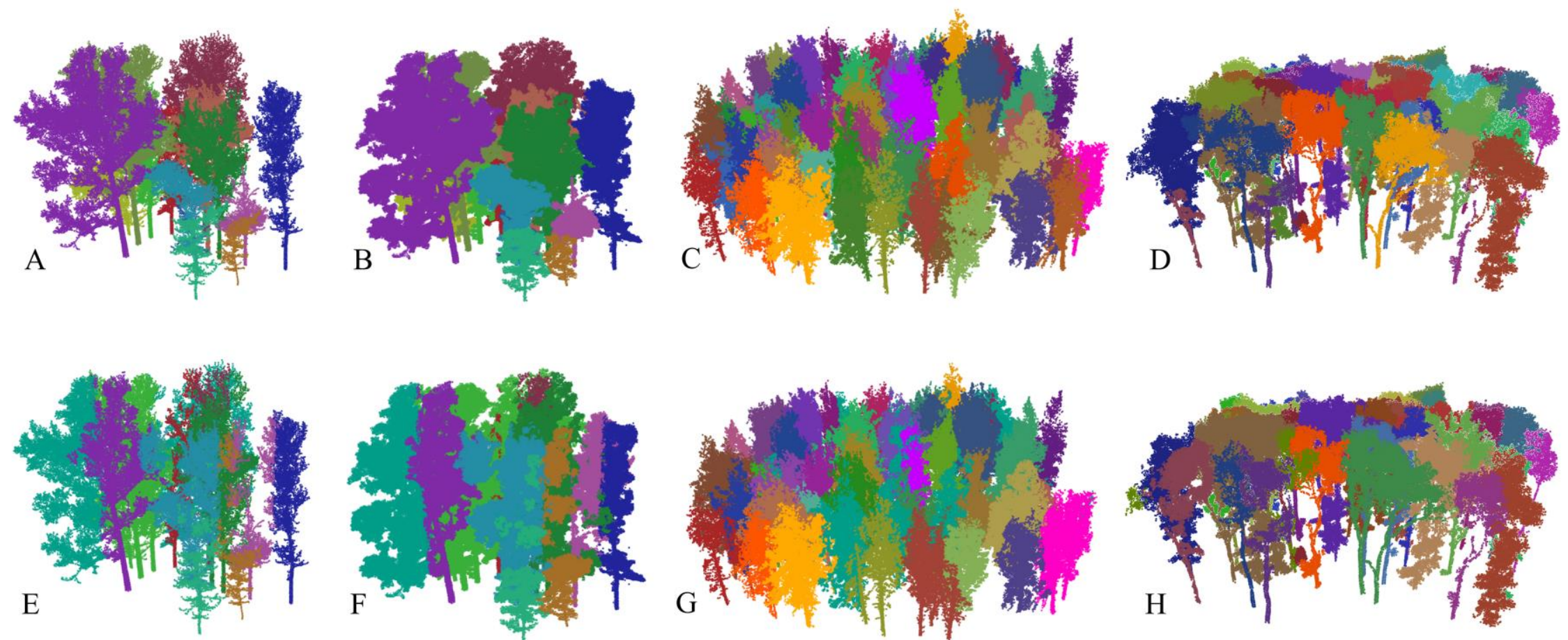

416 Figure 6. Trees segmented using the comparative shortest-path algorithm for Plot 1 during leaf-off condition (A), Plot 1 during leaf-on condition

417 (B), Plot 2 (C) and Plot 3 (D). E-H are the same plots segmented using PCS algorithm. Different colors indicate different trees. For quantitative

418 assessments on the segmentation results, see Table 3 and Figure 7. Please also see supplementary video for more details on the segmentation results. 
Table 3. Accuracy assessments of segmentation results at tree level. CSP stands for the comparative shortest-path algorithm and PCS stands for

421 the point cloud segmentation algorithm. For point-level assessments, see Figure 7.

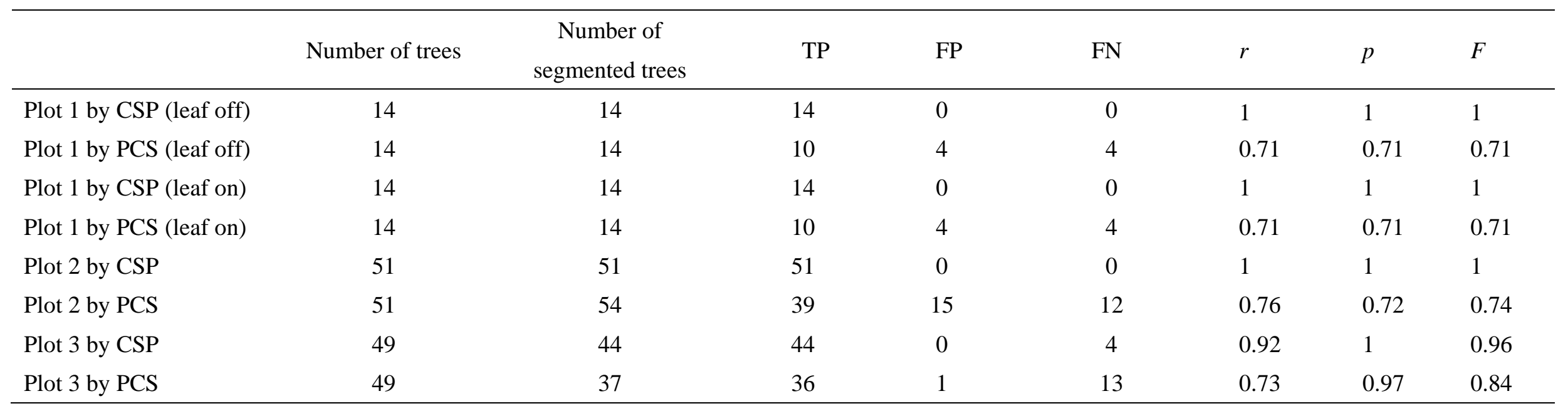



comparative shortest-path algorithm is high in terms of $r$ score, $p$ score, $F$ score and kappa

424 coefficients. The kappa coefficients for the comparative shortest-path algorithm ranged from 4250.83 to 0.93 , with the lowest value of 0.83 obtained in plot 3 and highest value of 0.93 in Plot 426 2 (Figure 7). The false rates ranged from $5 \%$ to $16 \%$ (Figure 7), and most falsely segmented 427 points were located at the outer-most parts of the crowns (Figure 6). Compared with the high 428 accuracy obtained using the comparative shortest-path algorithm, the results obtained using 429 PCS showed low accuracy, with kappa coefficients ranging from 0.30 to 0.65 and false rate 430 ranging from $33 \%$ to $64 \%$ (Figure 7 ). Despite the overall low accuracy, the segmentation 431 results by PCS for coniferous forest (i.e., Plot 3) were much better than broadleaved forest 432 (i.e., Plot 1 and Plot 3) (Figure 7). Totally $6.1 \%$ points and $33.9 \%$ points were falsely 433 segmented during leaf-off condition by the comparative shortest-path algorithm and PCS, 434 respectively, whereas the falsely segmented points represented $13.2 \%$ and $53.5 \%$, respectively, 435 during leaf-on condition (Figure 7). In summary, the segmentation results at both tree level 436 and point level demonstrate the superiority of the comparative shortest-path algorithm over 437 PCS. 

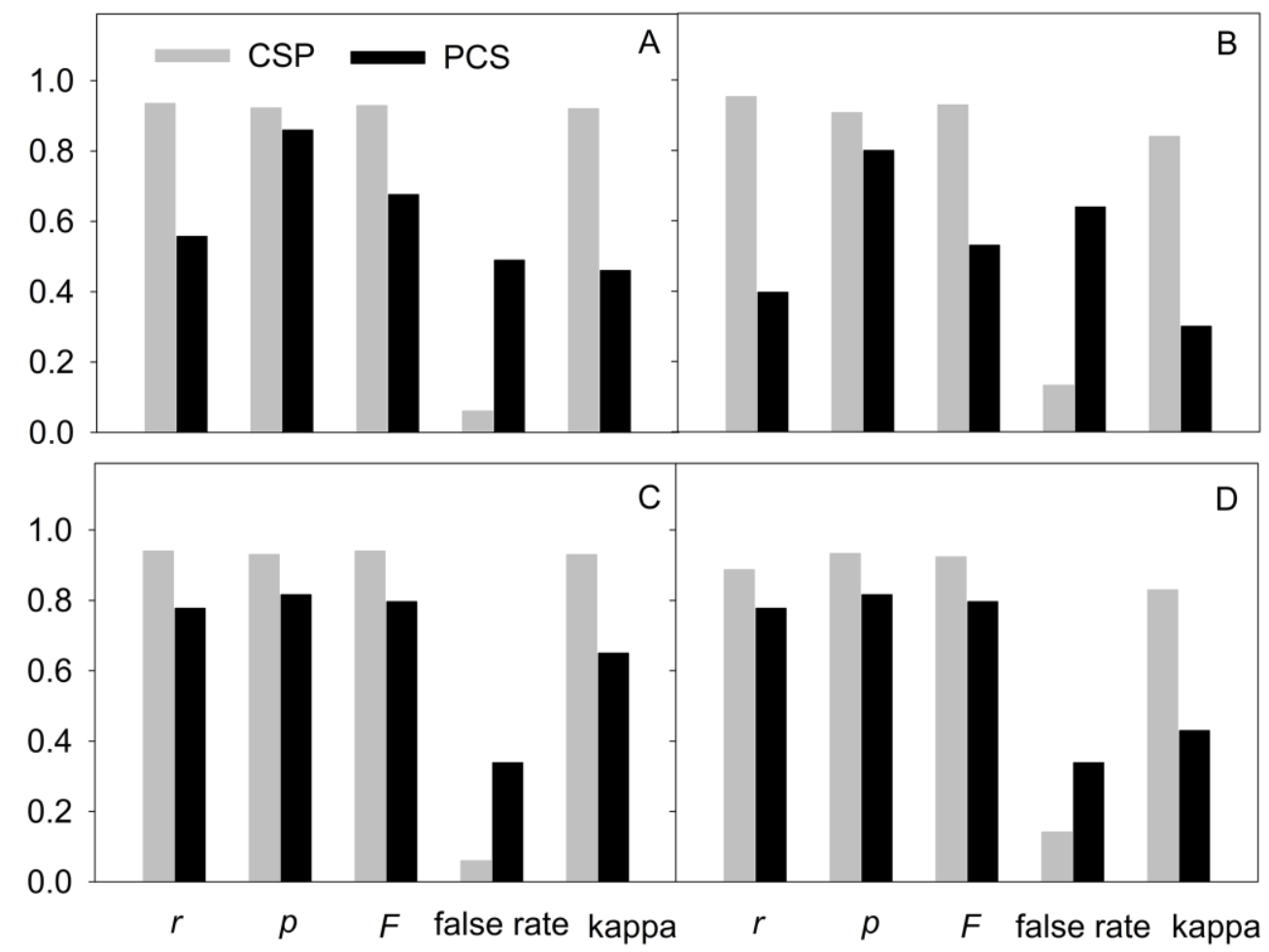

439 Figure 7. Point level accuracy assessments for Plot 1 during leaf-off condition (A), Plot 1 440 during leaf-on condition (B), Plot 2 (C) and Plot 3 (D). CSP stands for the comparative 441 shortest-path algorithm and PCS stands for the point cloud segmentation algorithm.

\section{Discussion}

5.1 Comparison of segmentation results obtained using comparative shortest-path algorithm and PCS

Both the comparative shortest-path algorithm and PCS produce high accuracy with respect to segmenting mobile-LiDAR-scanned roadside trees (Figure 5). Figure 4A clearly shows the ability of the comparative shortest-path algorithm to segment roadside trees. PCS is also expected to be successful because PCS seeks local maxima as seed points, and then grows the point cluster by judging a predefined horizontal spacing threshold. As long as the tree top is clearly presented and the spacing threshold is appropriately defined, PCS is able to segment 
individual trees accurately. In this study, the roadside trees were "naturally" separated from each other in space, which makes it easy to select the spacing threshold for PCS. Several methods have been proposed for segmenting roadside trees (Livny et al., 2010; Wu et al., 2013a). Previous research, together with the successes of the comparative shortest-path algorithm and PCS, lays a solid foundation for tree characteristic extraction using T-LiDAR and mobile LiDAR.

For T-LiDAR-scanned trees in forests with crown intersections, the comparative shortest-path algorithm shows high accuracy, even at the point level, and outperforms PCS (Figures 6 and 7). The segmentation results using the comparative shortest-path algorithm for Plot 1 and Plot 2 are better than that for Plot 3, because four trunks were not scanned in Plot 3 as a result of occlusion. The segmentation result for Plot 2 (coniferous) is better than that of Plot 1 and Plot 2 (both broadleaved) using PCS. PCS is originally designed to process airborne LiDAR data in coniferous forests; it is based on a 2D horizontal spacing threshold which cannot cope with horizontal crown-intersecting issues (Tao et al., 2014). As shown in Figure 6, a large number of shoots were misclassified by PCS, because of the irregular shapes of the tree crowns and the heavy intersection. In contrast, the comparative shortest-path algorithm is a bottom-up region-growing approach designed to process T-LiDAR data; it 469 classifies points one by one using the 3D path distance to the tree trunk instead of a constant $4702 \mathrm{D}$ spacing threshold to the tree set. The path distance is biologically meaningful, which helps 471 to allocate points at the intersecting region to the corresponding trees (Figure 8). The 472 superiority of CSP over PCS is therefore confirmed for ground-based LiDAR and for 473 segmenting broadleaved forest in this study. 


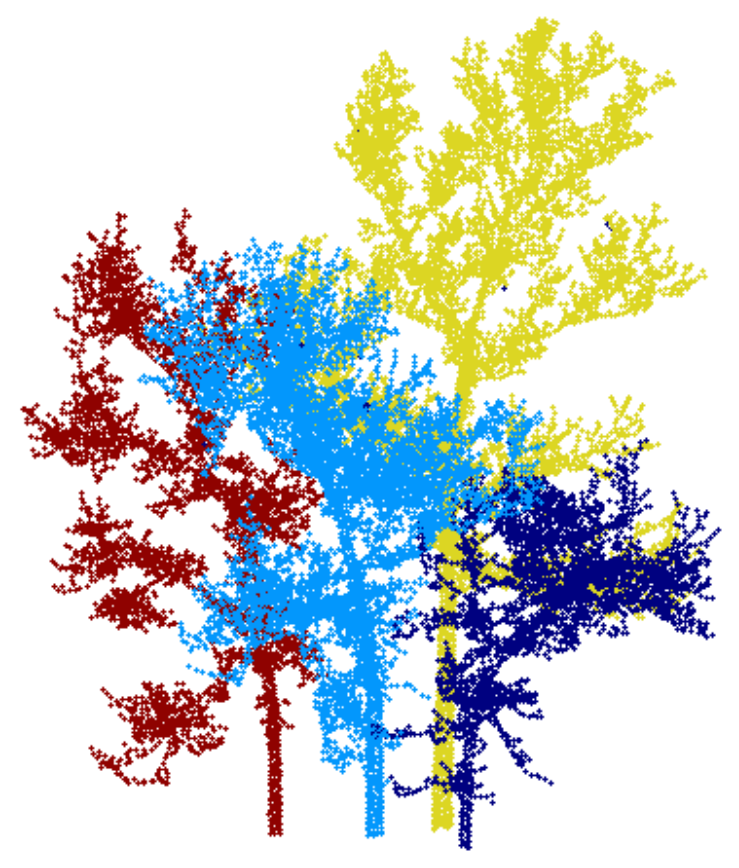

474

475 Figure 8. Details of the segmentation results for trees in Figure 1B, part of Plot 1. This figure 476 shows the comparative shortest-path algorithm is able to segment crowns with irregular 477 shapes.

\subsection{Comparison of segmentation results obtained during leaf-off and leaf-on conditions}

The kappa coefficient for the comparative shortest-path algorithm during leaf-off condition is 0.92, slightly higher than that of 0.84 during leaf-on condition. Similarly, the kappa coefficient for PCS during leaf-off condition is 0.46 , also higher than that of 0.30 during leaf-on condition. Meanwhile, the false rate for the leaf-off condition is much lower than that for leaf-on condition (Figure 7). According to the results obtained using both the comparative 485 shortest-path algorithm and PCS, the accuracy for leaf-off condition is higher than that for 486 leaf-on condition (Figure 7). Leaves greatly increase the point quantity and complicate crown 487 intersecting, making individual tree segmentation of the crown part more difficult, as 

shortest-path algorithm and $33.9 \%$ for PCS during leaf-off condition, whereas increased to $13.2 \%$ and $53.5 \%$, respectively, during leaf-on condition. For the comparative shortest-path algorithm, this phenomenon can be explained by the fact that the leaf clusters shade twigs and small branches, and this influences the correct extraction of the shortest path for some crown points.

\subsection{Parameters of comparative shortest-path algorithm}

Two parameters are optimized by the comparative shortest-path algorithm: MinPts for

DBSCAN and the search radius for building a graph among the cloud points. However, neither of these two parameters is sensitive to testing datasets and, furthermore, they can be set at fixed values provided same sampling setup. The first parameter, MinPts, of DBSCAN for detecting trunks, is determined directly from the point density. For a given T-LiDAR sampling setup, the point density at each trunk slice should be highly uniform (Cifuentes et al.,

501 2014). In addition, the distance between trunks is much greater than the average distance among LiDAR points; this also ensures the success of DBSCAN, because trunks "naturally" fall into separate clusters. The second parameter is the search radius for building a graph of

504 LiDAR-scanned points. This parameter is also directly determined by the LiDAR point 505 density. It is worth mentioning that DBSCAN was chosen for trunk segmentation not only 506 because of its accuracy, but also its ability to exclude noise points which might represent 507 shrubs. DBSCAN labels all points as core, border, or noise points: Given a point $p$, if the size 508 of $N_{\mathrm{Eps}}(p)$ is at least MinPts, then $p$ is a core point; if it is not a core point but $N_{\mathrm{Eps}}(p)$ contains 509 at least one core point, $p$ is a border point; otherwise, $p$ is treated as a noise point. In our case, 
511 DBSCAN. However, we acknowledge that if the input point slice for DBSCAN contains 512 shrub points which meet the standard of "core" or "border" points, DBSCAN is not able to 513 eliminate them. Thus, for a forest with complex under-canopy conditions, DBSCAN can be 514 replaced with circle-fitting procedures to identify circles directly from point slice (Tao et al., 515 2015). Our algorithm is being integrated into LiForest software (http://www.liforest.com) and 516 circle-fitting procedures will be available to exclude shrubs.

517 The comparative shortest-path algorithm requires the branch structure at the crown part to 518 be adequately scanned using T-LiDAR or mobile LiDAR, for calculating the correct shortest 519 paths for points at tree crowns. Otherwise, the distance of the shortest path from insufficiently 520 scanned branches might be falsely calculated, and the points at the corresponding sections 521 will be falsely classified. In Plot 3, four trees were falsely segmented because of trunk 522 occlusion (Table 3; Figure 6). This indicates that gaps within point clouds can influence the 523 segmentation accuracy of the comparative shortest-path algorithm. Meanwhile, the method 524 for DBH calculation might be biased towards underestimation at scanning from one direction

525 (Figure 3B). To maximize the expected utility of the comparative shortest-path algorithm, we 526 therefore recommend multi-scanning when field scanning is conducted. Meanwhile, a 527 searching probe for connecting the disconnected graph components will be integrated into the 528 proposed algorithm (Xu et al., 2007), to make it robust to occlusions. For forest plots in which 529 tree sizes are uniform, the normalization of conducting paths can also be omitted to reduce 530 computational burden.

\section{$531 \quad 5.4$ Ecological theories as guidelines to process LiDAR data}


The comparative shortest-path algorithm fully takes advantage of ecological basis and metabolic ecology theory as guidelines (Leopold, 1971; West et al., 1999a). Metabolic

534 ecology theory is a universal scaling law of tree growth and has strong empirical support

535 (Enquist, 2002; Bentley et al., 2013). This ensures that the proposed new algorithm might be 536 extensively applicable. Although there will always be trees deviating from the scaling 537 exponents of $2 / 3$, our segmentation results demonstrate that metabolic ecology theory can be 538 applied to segmenting broadleaved and coniferous forests (Figure 6 and supplementary video), 539 and relatively high accuracies can be achieved. It's been suggested that the use of metabolic 540 scaling theory should take factors like demographic traits and light into consideration (West et 541 al., 1999b; Bentley et al., 2013), and our future work will continue to explore these issues. In 542 fact, the effectiveness of metabolic ecology theory in assisting LiDAR data processing has 543 also been reported by previous researchers: Chen et al. (2007) estimated individual-tree basal 544 areas and stem volumes based on a recent advance in the metabolic ecology theory by Enquist 545 (2002). The results obtained by Chen et al. (2007) suggest that models built based on 546 allometry theory are the best in terms of accuracy and sensitivity to errors in tree crown 547 segmentation. Swetnam and Falk (2014) developed a variable-area local maxima algorithm 548 that incorporates the predictions of ecological metabolic scaling theory into segmentation of 549 individual trees, and found that the frequency of commission errors was greatly reduced. 550 These studies together with our segmentation algorithm suggest that the use of ecological 551 theories as guidelines for processing LiDAR data might improve the accuracy as well as the 552 efficiency. 


\section{Conclusion}

555 Forest scanned using T-LiDAR or mobile LiDAR are difficult to segment in terms of crown

556 segmentation. In this study, we developed a comparative shortest-path algorithm (CSP) with

557 the aim of segmenting both broadleaved and coniferous forests scanned using T-LiDAR and

558 roadside trees scanned using mobile LiDAR. The proposed algorithm shows high accuracy

559 not only in terms of the number of tree detected but also in terms of crown segmentation.

560 Visual inspection of the segmentation results suggested that high accuracy can be obtained,

561 with tree trunks successfully segmented from the point cloud but not missing details at the

562 crown parts. Furthermore, kappa coefficients at point levels ranging from 0.83 to 0.93 were

563 obtained for different testing dataset. A comparison with the PCS method reported by Li et al.

564 (2012) further demonstrated the superiority of CSP in segmenting broadleaved trees scanned 565 using T-LiDAR. The success of the proposed new algorithm provides a solid foundation for 566 our future research, where accurately segmented tree crowns will be analyzed to obtain 567 branch and leaf level forest characteristics, and finally quantify the branching network of 568 trees.

\section{Acknowledgments}

571 We thank Wenhan Cao for preparing Figure 4, and Shuxin Pang, Linhai Chen, Yongyi Zhu 572 and Xin Yu for their assistance in LiDAR scanning. We also thank the reviewers for their 573 constructive comments and recommendations. This work was partly supported by the

574 National Natural Science Foundation of China (\#31321061, 31330012, 41471363 and $57541401505)$. 


\section{References}

Asner, G.P., Powell, G.V.N., Mascaro, J., Knapp, D.E., Clark, J.K., Jacobson, J., Kennedy-Bowdoin, T., Balaji, A., Paez-Acosta, G., Victoria, E., Secada, L., Valqui, M., Hughes, R.F., 2010. High-resolution forest carbon stocks and emissions in the Amazon. Proc Natl Acad Sci USA 107(28), 16738-16742.

Bentley, L.P., Stegen, J.C., Savage, V.M., Smith, D.D., von Allmen, E.I., Sperry, J.S., Reich, P.B., Enquist, B.J., 2013. An empirical assessment of tree branching networks and implications for plant allometric scaling models. Ecol Lett 16(8), 1069-1078.

Bienert, A., Scheller, S., Keane, E., Mullooly, G., Mohan, F., 2006. Application of terrestrial laser scanners for the determination of forest inventory parameters. Int Arch Photogramm Remote Sens Spatial Inf Sci 36 (Part 5).

Brolly, G., Kiraly, G., 2009. Algorithms for stem mapping by means of terrestrial laser scanning. Acta Silvatica et Lignaria Hungarica 5, 119-130.

Chen, Q., Baldocchi, D., Gong, P., Kelly, M., 2006. Isolating individual trees in a savanna woodland using small footprint LiDAR data. Photogramm Eng Rem S 72(8), 923-932.

Chen, Q., Gong, P., Baldocchi, D., Tian, Y.Q., 2007. Estimating basal area and stem volume for individual trees from LiDAR data. Photogramm Eng Rem S 73(12), 1355-1365.

Cifuentes, R., Van der Zande, D., Farifteh, J., Salas, C., Coppin, P., 2014. Effects of voxel size and sampling setup on the estimation of forest canopy gap fraction from terrestrial laser scanning data. Agr Forest Meteorol 194, 230-240.

Cormen, T. H., Leiserson, C. E., Rivest, R. L., Stein, C., 2009. Introduction to algorithms, third ed. Vol. 6, pp. 643. Cambridge: MIT press. 
Dassot, M., Colin, A., Santenoise, P., Fournier, M., Constant, T., 2012. Terrestrial laser scanning for measuring the solid wood volume, including branches, of adult standing trees in the forest environment. Comput Electron Agr 89, 86-93.

Dassot, M., Constant, T., Fournier, M., 2011. The use of terrestrial LiDAR technology in forest science: application fields, benefits and challenges. Ann For Sci 68(5), 959-974.

Daszykowski, M., Walczak, B., Massart, D.L., 2002. Looking for natural patterns in analytical data. 2. Tracing local density with OPTICS. J Chem Inf Comp Sci 42(3), $500-507$.

Dijkstra, E.W., 1959. A note on two problems in connexion with graphs. Numer Math 1, 269-271.

Ester, M., Kriegel, H. P., Sander, J., Xu, X., 1996. A density-based algorithm for discovering clusters in large spatial databases with noise, August. In Kdd, Vol. 96, pp. 226-231.

Enquist, B.J., 2002. Universal scaling in tree and vascular plant allometry: Toward a general quantitative theory linking plant form and function from cells to ecosystems. Tree Physiol 22(15-16), 1045-1064.

Gorte, B., Pfeifer, N., 2004. Structuring laser-scanned trees using 3D mathematical morphology. International Archives of Photogrammetry and Remote Sensing 35, 929-933.

Goutte, C., Gaussier, E., 2005. A probabilistic interpretation of precision, recall and F-score, with implication for evaluation. Lect Notes Comput Sc 3408, 345-359. 
619 Guo, Q.H., Li, W.K., Yu, H., Alvarez, O., 2010. Effects of topographic variability and

620 LiDAR sampling density on several DEM interpolation methods. Photogramm Eng Rem $621 \quad$ S 76(6), 701-712.

622 Haralick, R.M., 1974. A measure of circularity of digital figures. IEEE Trans. Systems Man $623 \quad$ Cybernet. 4(4), 394-396.

624 Huang, H.B., Li, Z., Gong, P., Cheng, X.A., Clinton, N., Cao, C.X., Ni, W.J., Wang, L., 2011. 625 Automated methods for measuring DBH and tree heights with a commercial scanning 626 LiDAR. Photogramm Eng Rem S 77(3), 219-227.

627

Hyyppa, J., Kelle, O., Lehikoinen, M., Inkinen, M., 2001. A segmentation-based method to 628 retrieve stem volume estimates from 3-D tree height models produced by laser scanners. Ieee T Geosci Remote 39(5), 969-975.

Hyyppa, J., Yu, X.W., Hyyppa, H., Vastaranta, M., Holopainen, M., Kukko, A., Kaartinen, H., Jaakkola, A., Vaaja, M., Koskinen, J., Alho, P., 2012. Advances in forest inventory using 632 airborne laser scanning. Remote Sens-Basel 4(5), 1190-1207.

Kaartinen, H., Hyyppa, J., Yu, X.W., Vastaranta, M., Hyyppa, H., Kukko, A., Holopainen, M., 634 Heipke, C., Hirschmugl, M., Morsdorf, F., Naesset, E., Pitkanen, J., Popescu, S., Solberg, 635 636 S., Wolf, B.M., Wu, J.C., 2012. An International comparison of individual tree detection terrestrial laser scanning. J Appl Remote Sens 2. 
641 Li, L., Guo, Q.H., Tao S.L., Kelly, M., Xu, G.C., 2015. Lidar with multi-temporal MODIS provide a means to upscale predictions of forest biomass. Isprs $\mathrm{J}$ Photogramm 643 102:198-208.

644

Li, W. K., Guo, Q. H., Jakubowski, M.K., Kelly, M., 2012. A new method for segmenting individual trees from the LiDAR point cloud. Photogramm Eng Rem S 78(1), 75-84.

Livny, Y., Yan, F.L., Olson, M., Chen, B.Q., Zhang, H., El-Sana, J., 2010. Automatic reconstruction of tree skeletal structures from point clouds. Acm T Graphic 29(6).

Lovell, J.L., Jupp, D.L.B., Newnham, G.J., Culvenor, D.S., 2011. Measuring tree stem diameters using intensity profiles from ground-based scanning LiDAR from a fixed viewpoint. Isprs J Photogramm 66(1), 46-55.

Maas, H.G., Bienert, A., Scheller, S., Keane, E., 2008. Automatic forest inventory parameter determination from terrestrial laser scanner data. Int J Remote Sens 29(5), 1579-1593.

653 Maltamo, M., Eerikainen, K., Pitkanen, J., Hyyppa, J., Vehmas, M., 2004. Estimation of 654 timber volume and stem density based on scanning laser altimetry and expected tree size distribution functions. Remote Sens Environ 90(3), 319-330. classification and identification of tree stems using ground-based LiDAR. J Field Robot 29(6), 891-910.

659 Moorthy, I., Miller, J.R., Berni, J.A.J., Zarco-Tejada, P., Hu, B.X., Chen, J., 2011. Field 660 characterization of olive (Olea europaea L.) tree crown architecture using terrestrial laser scanning data. Agr Forest Meteorol 151(2), 204-214. 
Naesset, E., 1997. Determination of mean tree height of forest stands using airborne laser scanner data. Isprs J Photogramm 52(2), 49-56.

Olofsson, K., Holmgren, J., Olsson, H., 2014. Tree stem and height measurements using Terrestrial Laser Scanning and the RANSAC algorithm. Remote Sens 6(5), 4323-4344.

Popescu, S.C., Wynne, R.H., Nelson, R.F., 2002. Estimating plot-level tree heights with LiDAR: local filtering with a canopy-height based variable window size. Comput Electron Agr 37(1-3), 71-95.

Reitberger, J., Schnorr, C., Krzystek, P., Stilla, U., 2009. 3D segmentation of single trees exploiting full waveform LIDAR data. Isprs J Photogramm 64(6), 561-574.

Schilling, A., Schmidt, A., Maas, H. G. 2011. Automatic Tree Detection and Diameter Estimation in Terrestrial Laser Scanner Point Clouds. Proceedings of 16th Computer Vision Winter Workshop, February, pp. 75-83.

Schilling, A., Schmidt, A., Maas, H.G., 2012. Tree topology representation from TLS point clouds using depth-first search in voxel space. Photogramm Eng Rem S 78(4), 383-392.

Siek, J.G., Lee, L.-Q., Lumsdaine, A., 2001. Boost Graph Library: User Guide and Reference Manual. Pearson Education.

Simonse, M., Aschoff, T., Spiecker, H., Thies, M., 2003. Automatic determination of forest inventory parameters using terrestrial laser scanning. Proceedings of the Scandlaser Scientific Workshop on Airborne Laser Scanning of Forests, Umea, Sweden, 3-4 September, pp. 251-257.

Sokolova, M., N. Japkowicz, S. Szpakowicz, 2006. Beyondaccuracy, F-score and ROC: A family of discriminant measures for performance evaluation, AI 2006: Advances in 
684

685

686

687

688

689

690

691

692

693

694

695

696

697

698

699

700

701

702

703

704

705

Artificial Intelligence (A. Sattar and B.-H. Kang, editors), Springer Berlin, Heidelberg, pp. 1015-1021.

Swetnam, T.L., Falk, D.A., 2014. Application of metabolic scaling theory to reduce error in local maxima tree segmentation from aerial LiDAR. Forest Ecol Manag 323, 158-167.

Tao, S.L., Guo, Q.H., Xu, S.W., Su, Y.J., Li, Y.M., Wu, F.F., 2015. A Geometric Method for Wood-Leaf Separation Using Terrestrial and Simulated LiDAR Data. Photogramm Eng Rem S 81(10), 767-776.

Tao, S.L., Guo, Q.H., Li, L., Xue, B.L., Kelly, M., Li, W.K., Xu, G.C., Su, Y.J., 2014. Airborne Lidar-derived volume metrics for aboveground biomass estimation: A comparative assessment for conifer stands. Agr Forest Meteorol 198, $24-32$.

Wehr, A., Lohr, U., 1999. Airborne laser scanning - an introduction and overview. Isprs J Photogramm 54(2-3), 68-82.

West, G.B., Brown, J.H., Enquist, B.J., 1999a. The fourth dimension of life: Fractal geometry and allometric scaling of organisms. Science 284(5420), 1677-1679.

West, G.B., Brown, J.H., Enquist, B.J., 1999b. A general model for the structure and allometry of plant vascular systems. Nature 400(6745), 664-667.

Wu, B., Yu, B.L., Yue, W.H., Shu, S., Tan, W.Q., Hu, C.L., Huang, Y., Wu, J.P., Liu, H.X., 2013a. A voxel-based method for automated identification and morphological parameters estimation of individual street trees from mobile laser scanning data. Remote Sens-Basel 5(2), 584-611.

Wu, J.Y., Cawse-Nicholson, K., van Aardt, J., 2013b. 3D Tree reconstruction from simulated small footprint waveform LiDAR. Photogramm Eng Rem S 79(12), 1147-1157. 
706 Xu, H., Gossett, N., Chen, B.Q., 2007. Knowledge and heuristic-based modeling of $707 \quad$ laser-scanned trees. Acm T Graphic 26(4).

708 Yao, W., Krzystek, P., Heurich, M., 2012. Tree species classification and estimation of stem 709 volume and DBH based on single-tree extraction by exploiting airborne full-waveform $710 \quad$ LiDAR data. Remote Sens Environ 123, 368-380.

711 Zhao, K.G., Popescu, S., Nelson, R., 2009. Lidar remote sensing of forest biomass: A 712 scale-invariant estimation approach using airborne lasers. Remote Sens Environ 113(1), $713 \quad 182-196$.

714 Zheng, G., Moskal, L.M., Kim, S.H., 2013. Retrieval of effective leaf area index in 715 heterogeneous forests with terrestrial laser scanning. Ieee T Geosci Remote 51(2), $716 \quad 777-78$ 